\title{
A short proof of generalized Jacobi-Trudi expansions for Macdonald polynomials
}

\author{
Michel Lassalle \\ Centre National de la Recherche Scientifique \\ Institut Gaspard Monge, Université de Marne-la-Vallée \\ 77454 Marne-la-Vallée Cedex, France \\ lassalle@univ-mlv.fr \\ http://igm.univ-mlv.fr/ lassalle
}

January 5, 2004

\begin{abstract}
We give an elementary proof of the development of Macdonald polynomials in terms of "modified complete" and elementary symmetric functions.
\end{abstract}

\section{Introduction}

In spite of many efforts, the problem of finding an explicit analytic expansion for the "zonal polynomials" of Hua [1] and James [2] has kept unsolved for fifty years. This problem is now better understood in the more general framework of Macdonald polynomials [12]. Zonal polynomials are indeed a special case of Jack polynomials, which are themselves a particular limit of Macdonald polynomials.

Macdonald polynomials form a basis of the algebra of symmetric functions with rational coefficients in two parameters $q, t$. They generalize several classical bases of this algebra, including monomial, elementary, Schur, Hall-Littlewood, and Jack symmetric functions. These particular cases correspond to various specializations of the indeterminates $q$ and $t$.

Although two combinatorial expansions are known for Macdonald polynomials, in terms of tableaux [12 or determinants [7, no analytic expansion was available for them. Such a development had only been worked out when the indexing partition is a hook [4, has length two [3] or three [8, and in the dual cases corresponding to parts at most equal to 3 . 
In [10] a general solution has been announced for this problem, providing two explicit analytic developments for Macdonald polynomials, in terms of elementary and "modified complete" symmetric functions. In the special case $q=t$, these two developments coincide with the classical Jacobi-Trudi formulas for Schur functions. Thus the results announced in [10] appear as generalized Jacobi-Trudi expansions for Macdonald polynomials.

The purpose of this paper is to give an elementary proof of this result. A completely different proof has been reported in another paper [11. This other proof is actually the first one in chronological order. It relies on the Pieri formula for Macdonald polynomials. This Pieri formula defines an infinite multidimensional matrix which may be explicitly inverted. This inversion is performed using a method originally developed by Krattenthaler [5. 6], and further adapted to the multivariate case by Schlosser [13].

Both proofs have their own advantages. On the one hand, the inversion of the "Pieri matrix" was the natural problem to solve, and it was needed for bringing to light the explicit form of the solution. However this method is rather technical and essentially external to Macdonald's theory. On the other hand, the proof given here is very quick and elementary, it does not use the general Pieri formula and keeps totally inside the Macdonald framework.

A strange fact is that our proof studies Macdonald polynomials $P_{\lambda}$, with $\lambda$ a partition of length $n$, by applying Macdonald theory to some auxiliary space of $n$ indeterminates $u_{i}, 1 \leq i \leq n$, specialized to $u_{i}=q^{\lambda_{i}-\lambda_{n}} t^{n-i-1}$. It is an open question whether an analogous method can be applied to Macdonald polynomials associated with other root systems than $A_{n}$.

\section{Macdonald polynomials}

The standard reference for Macdonald polynomials is Chapter 6 of [12].

A partition $\lambda=\left(\lambda_{1}, \ldots, \lambda_{n}\right)$ is a finite weakly decreasing sequence of nonnegative integers, called parts. The number $l(\lambda)$ of positive parts is called the length of $\lambda$, and $|\lambda|=\sum_{i=1}^{n} \lambda_{i}$ the weight of $\lambda$. For any integer $i \geq 1, m_{i}(\lambda)=\operatorname{card}\left\{j: \lambda_{j}=i\right\}$ is the multiplicity of the part $i$ in $\lambda$. Clearly $l(\lambda)=\sum_{i \geq 1} m_{i}(\lambda)$ and $|\lambda|=\sum_{i \geq 1} i m_{i}(\lambda)$. We shall also write $\lambda=\left(1^{m_{1}}, 2^{m_{2}}, 3^{m_{3}}, \ldots\right)$. We set

$$
z_{\lambda}=\prod_{i \geq 1} i^{m_{i}(\lambda)} m_{i}(\lambda) !
$$

We denote $\lambda^{\prime}$ the partition conjugate to $\lambda$, whose parts are given by $m_{i}\left(\lambda^{\prime}\right)=\lambda_{i}-\lambda_{i+1}$. We have $\lambda_{i}^{\prime}=\sum_{j \geq i} m_{j}(\lambda)$.

Let $X=\left\{x_{1}, x_{2}, x_{3}, \ldots\right\}$ be a (finite or infinite) set of indeterminates and $\mathcal{S}$ the corresponding algebra of symmetric functions with coefficients in $\mathrm{Q}$. When $X$ is infinite, elementary symmetric functions $e_{k}(X)$, complete symmetric functions $h_{k}(X)$, power sum symmetric functions $p_{k}(X)$ form three algebraic bases of $\mathcal{S}$, which can thus be viewed as an abstract algebra over $\mathrm{Q}$ generated by the functions $e_{k}, h_{k}$ or $p_{k}$. 
Let $q, t$ be two indeterminates. We define $(a ; q)_{0}=1$ and for $k \geq 1$,

$$
(a ; q)_{k}=\prod_{i=0}^{k-1}\left(1-a q^{i}\right), \quad(a ; q)_{\infty}=\prod_{i \geq 0}\left(1-a q^{i}\right) .
$$

Let $\mathrm{Q}[q, t]$ be the field of rational functions in $q, t$, and Sym $=\mathcal{S} \otimes \mathrm{Q}[q, t]$ the algebra of symmetric functions with coefficients in $\mathrm{Q}[q, t]$.

For any $k \geq 0$, the "modified complete" symmetric function $g_{k}(X ; q, t)$ is defined by the generating series

$$
\prod_{i \geq 1} \frac{\left(t u x_{i} ; q\right)_{\infty}}{\left(u x_{i} ; q\right)_{\infty}}=\sum_{k \geq 0} u^{k} g_{k}(X ; q, t) .
$$

It is often written in $\lambda$-ring notation [9, p. 223], i.e.

$$
g_{k}(X ; q, t)=h_{k}\left[\frac{1-t}{1-q} X\right] .
$$

The symmetric functions $g_{k}(q, t)$ form an algebraic basis of Sym, which may be expanded in terms of any classical basis. This development is explicitly given in [12, pp. 311 and 314] in terms of power sums and monomial symmetric functions, and in [9, Sect. 10, p. 237] in terms of other classical bases.

We now restrict to the case of a finite set of indeterminates $X=\left\{x_{1}, \ldots, x_{n}\right\}$. Let $T_{q, x_{i}}$ denote the $q$-deformation operator defined by

$$
T_{q, x_{i}} f\left(x_{1}, \ldots, x_{n}\right)=f\left(x_{1}, \ldots, q x_{i}, \ldots, x_{n}\right)
$$

and for any $1 \leq i \leq n$,

$$
A_{i}(X ; t)=\prod_{\substack{k=1 \\ k \neq i}}^{n} \frac{t x_{i}-x_{k}}{x_{i}-x_{k}}
$$

Macdonald polynomials $P_{\lambda}(X ; q, t)$, with $\lambda$ a partition such that $l(\lambda) \leq n$, are defined as the eigenvectors of the following difference operator

$$
E(q, t)=\sum_{i=1}^{n} A_{i}(X ; t) T_{q, x_{i}}
$$

One has

$$
E(q, t) P_{\lambda}(X ; q, t)=\left(\sum_{i=1}^{n} q^{\lambda_{i}} t^{n-i}\right) P_{\lambda}(X ; q, t) .
$$

More generally let $\Delta(X)=\prod_{1 \leq i<j \leq n}\left(x_{i}-x_{j}\right)$ be the Vandermonde determinant and $a$ some indeterminate. Macdonald polynomials $P_{\lambda}(X ; q, t)$ are eigenvectors of the difference operator

$$
D(a ; q, t)=\frac{1}{\Delta(X)} \operatorname{det}_{1 \leq i, j \leq n}\left[x_{i}^{n-j}\left(1+a t^{n-j} T_{q, x_{i}}\right)\right]
$$


One has

$$
D(a ; q, t) P_{\lambda}(X ; q, t)=\prod_{i=1}^{n}\left(1+a q^{\lambda_{i}} t^{n-i}\right) P_{\lambda}(X ; q, t) .
$$

The polynomials $P_{\lambda}(X ; q, t)$ form an orthogonal basis of Sym with respect to the scalar product $<,>_{q, t}$ defined by

$$
<p_{\lambda}, p_{\mu}>_{q, t}=\delta_{\lambda \mu} z_{\lambda} \prod_{i=1}^{l(\lambda)} \frac{1-q^{\lambda_{i}}}{1-t^{\lambda_{i}}}
$$

where $p_{\mu}$ denotes the power sum symmetric function $p_{\mu}=\prod_{k=1}^{l(\mu)} p_{\mu_{k}}$. Let $Q_{\lambda}(X ; q, t)$ denote the dual basis of $P_{\lambda}(X ; q, t)$ for this scalar product.

Equivalently if $Y=\left\{y_{1}, \ldots, y_{n}\right\}$ is another set of $n$ indeterminates, and

$$
\Pi(X, Y ; q, t)=\prod_{i, j=1}^{n} \frac{\left(t x_{i} y_{j} ; q\right)_{\infty}}{\left(x_{i} y_{j} ; q\right)_{\infty}}
$$

we have

$$
\Pi(X, Y ; q, t)=\sum_{\lambda} P_{\lambda}(X ; q, t) Q_{\lambda}(Y ; q, t) .
$$

This yields immediately

$$
\begin{gathered}
D(a ; q, t)_{(X)} \Pi(X, Y ; q, t)=D(a ; q, t)_{(Y)} \Pi(X, Y ; q, t), \\
E(q, t)_{(X)} D(a ; q, t)_{(X)} \Pi(X, Y ; q, t)=E(q, t)_{(Y)} D(a ; q, t)_{(Y)} \Pi(X, Y ; q, t),
\end{gathered}
$$

where the suffix $X$ (resp. $Y$ ) indicates operation on the $X$ (resp. $Y$ ) variables. Now one has $P_{\lambda}(1 / q, 1 / t)=P_{\lambda}(q, t)$ and $Q_{\lambda}(1 / q, 1 / t)=\left(q t^{-1}\right)^{|\lambda|} Q_{\lambda}(q, t)$, which implies

$$
E(1 / q, 1 / t)_{(X)} D(a ; q, t)_{(X)} \Pi(X, Y ; q, t)=E(1 / q, 1 / t)_{(Y)} D(a ; q, t)_{(Y)} \Pi(X, Y ; q, t) .
$$

The Macdonald polynomials associated with a row or a column partition are given by

$$
P_{1^{k}}(q, t)=e_{k}, \quad Q_{(k)}(q, t)=g_{k}(q, t) .
$$

In the following, when the parameters $q, t$ are omitted, $P_{\mu}$ or $Q_{\mu}$ stands for $P_{\mu}(q, t)$ or $Q_{\mu}(q, t)$.

\section{Statement of results}

Let $\mathrm{N}$ the set of nonnegative integers. Let $u=\left(u_{1}, \ldots, u_{n}\right)$ be $n$ indeterminates and $\theta=\left(\theta_{1}, \ldots, \theta_{n}\right) \in \mathbf{N}^{n}$. For clarity of notations, we introduce $n$ auxiliary variables $v=$ 
$\left(v_{1}, \ldots, v_{n}\right)$ defined by $v_{k}=q^{\theta_{k}} u_{k}$. We write

$$
\begin{array}{r}
C_{\theta_{1}, \ldots, \theta_{n}}^{(q, t)}\left(u_{1}, \ldots, u_{n}\right)=\prod_{k=1}^{n} t^{\theta_{k}} \frac{(q / t ; q)_{\theta_{k}}}{(q ; q)_{\theta_{k}}} \frac{\left(q u_{k} ; q\right)_{\theta_{k}}}{\left(q t u_{k} ; q\right)_{\theta_{k}}} \prod_{1 \leq i<j \leq n} \frac{\left(q u_{i} / t u_{j} ; q\right)_{\theta_{i}}}{\left(q u_{i} / u_{j} ; q\right)_{\theta_{i}}} \frac{\left(t u_{i} / v_{j} ; q\right)_{\theta_{i}}}{\left(u_{i} / v_{j} ; q\right)_{\theta_{i}}} \\
\times \frac{1}{\Delta(v)} \operatorname{det}_{1 \leq i, j \leq n}\left[v_{i}^{n-j}\left(1-t^{j-1} \frac{1-t v_{i}}{1-v_{i}} \prod_{k=1}^{n} \frac{u_{k}-v_{i}}{t u_{k}-v_{i}}\right)\right] .
\end{array}
$$

Setting $u_{n+1}=1 / t$ we have

$$
\begin{aligned}
& C_{\theta_{1}, \ldots, \theta_{n}}^{(q, t)}\left(u_{1}, \ldots, u_{n}\right)= \prod_{1 \leq i<j \leq n+1} \frac{\left(q u_{i} / t u_{j} ; q\right)_{\theta_{i}}}{\left(q u_{i} / u_{j} ; q\right)_{\theta_{i}}} \prod_{1 \leq i \leq j \leq n} \frac{\left(t u_{i} / v_{j} ; q\right)_{\theta_{i}}}{\left(u_{i} / v_{j} ; q\right)_{\theta_{i}}} \\
& \times \frac{1}{\Delta(v)} \operatorname{det}_{1 \leq i, j \leq n}\left[v_{i}^{n-j}\left(1-t^{j} \prod_{k=1}^{n+1} \frac{u_{k}-v_{i}}{t u_{k}-v_{i}}\right)\right] .
\end{aligned}
$$

The following results were announced in [10] and proved in [11].

Theorem 1. Let $\lambda=\left(\lambda_{1}, \ldots, \lambda_{n+1}\right)$ be an arbitrary partition with length $n+1$. For any $1 \leq k \leq n+1$ define $u_{k}=q^{\lambda_{k}-\lambda_{n+1}} t^{n-k}$. We have

$$
Q_{\left(\lambda_{1}, \ldots, \lambda_{n+1}\right)}=\sum_{\theta \in \mathbb{N}^{n}} C_{\theta_{1}, \ldots, \theta_{n}}^{(q, t)}\left(u_{1}, \ldots, u_{n}\right) Q_{\left(\lambda_{n+1}-|\theta|\right)} Q_{\left(\lambda_{1}+\theta_{1}, \ldots, \lambda_{n}+\theta_{n}\right)} .
$$

There exists an automorphism $\omega_{q, t}=\omega_{t, q}{ }^{-1}$ of Sym such that

$$
\omega_{q, t}\left(Q_{\lambda}(q, t)\right)=P_{\lambda^{\prime}}(t, q), \quad \omega_{q, t}\left(g_{k}(q, t)\right)=e_{k} .
$$

Applying $\omega_{q, t}$ to Theorem 1, we obtain the following equivalent result.

Theorem 2. Let $\lambda=\left(1^{m_{1}}, 2^{m_{2}}, \ldots,(n+1)^{m_{n+1}}\right)$ be an arbitrary partition consisting of parts at most equal to $n+1$. For any $1 \leq k \leq n+1$ define $u_{k}=q^{n-k} t^{\sum_{j=k}^{n} m_{j}}$. We have

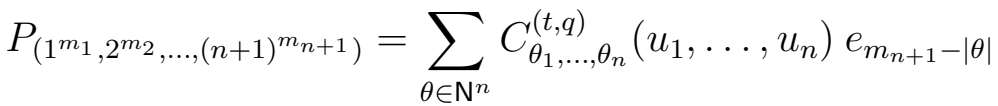

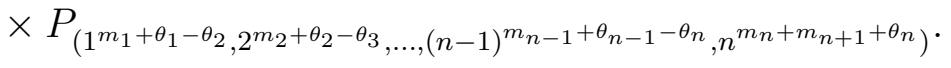

The specialization $q=t$ corresponds to the case of Schur functions. Then we have $P_{\lambda}(t, t)=Q_{\lambda}(t, t)=s_{\lambda}$ and $g_{k}(t, t)=h_{k}$. It may be shown [11, Sect. 7] that Theorem 11 reads

$$
s_{\left(\lambda_{1}, \ldots, \lambda_{n+1}\right)}=\sum_{\theta \in(0,1)^{n}}(-1)^{|\theta|} h_{\lambda_{n+1}-|\theta|} s_{\left(\lambda_{1}+\theta_{1}, \ldots, \lambda_{n}+\theta_{n}\right)} .
$$

This is exactly the development of the Jacobi-Trudi determinant

$$
s_{\lambda}=\operatorname{det}_{1 \leq i, j \leq n+1}\left[h_{\lambda_{i}-i+j}\right]
$$


along its last row [12, p. 41, equ. (3.4)]. Conversely Theorem 2 is exactly the development of the Nägelsbach-Kotska determinant

$$
s_{\lambda}=\operatorname{det}_{1 \leq i, j \leq n+1}\left[e_{\lambda_{i}^{\prime}-i+j}\right]
$$

along its last row. Our results thus appear as generalized Jacobi-Trudi expansions for Macdonald polynomials.

Let $\mathrm{M}^{(n)}$ denote the set of upper triangular $n \times n$ matrices with nonnegative integers, and 0 on the diagonal. By a straightforward iteration of Theorem 1 we deduce immediately the analytic development of Macdonald polynomials in terms of the symmetric functions $g_{k}$.

Theorem 3. Let $\lambda=\left(\lambda_{1}, \ldots, \lambda_{n+1}\right)$ be an arbitrary partition with length $n+1$. We have

$$
\begin{array}{r}
Q_{\lambda}(q, t)=\sum_{\theta \in \mathrm{M}^{(n+1)}} \prod_{k=1}^{n} C_{\theta_{1, k+1}, \ldots, \theta_{k, k+1}}^{(q, t)}\left(\left\{u_{i}=q^{\lambda_{i}-\lambda_{k+1}+\sum_{j=k+2}^{n+1}\left(\theta_{i, j}-\theta_{k+1, j}\right)} t^{k-i} ; 1 \leq i \leq k\right\}\right) \\
\times \prod_{k=1}^{n+1} g_{\lambda_{k}+\sum_{j=k+1}^{n+1} \theta_{k j}-\sum_{j=1}^{k-1} \theta_{j k}} .
\end{array}
$$

This result may also be stated in terms of "raising operators" [12, p. 9]. For each pair of integers $1 \leq i<j \leq n+1$ define an operator $R_{i j}$ acting on multi-integers $a=\left(a_{1}, \ldots, a_{n+1}\right)$ by $R_{i j}(a)=\left(a_{1}, \ldots, a_{i}+1, \ldots, a_{j}-1, \ldots, a_{n+1}\right)$. Any product $R=$ $\prod_{i<j} R_{i j}^{\theta_{i j}}$, with $\theta=\left(\theta_{i j}\right)_{1 \leq i<j \leq n+1} \in \mathrm{M}^{(n+1)}$ is called a raising operator. Its action may be extended to any function $g_{\mu}=\prod_{k=1}^{n+1} g_{\mu_{k}}$, with $\mu$ a partition of length $n+1$, by setting $R g_{\mu}=g_{R(\mu)}$. In particular $R_{i j} g_{\mu}=g_{\mu_{1}} \ldots g_{\mu_{i}+1} \ldots g_{\mu_{j}-1} \ldots g_{\mu_{n+1}}$. Then the quantity appearing above in the right-hand side may be written

$$
\prod_{k=1}^{n+1} g_{\lambda_{k}+\sum_{j=k+1}^{n+1} \theta_{k j}-\sum_{j=1}^{k-1} \theta_{j k}}=\left(\prod_{1 \leq i<j \leq n+1} R_{i j}^{\theta_{i j}}\right) g_{\lambda} .
$$

For $t=q$ we recover the following variant of the Jacobi-Trudi expansion ([12, p. 42)

$$
s_{\lambda}=\left(\prod_{1 \leq i<j \leq n+1}\left(1-R_{i j}\right)\right) h_{\lambda}
$$

Applying $\omega_{q, t}$, we immediatly deduce the following analytic expansion of Macdonald polynomials in terms of elementary symmetric functions $e_{k}$.

Theorem 4. Let $\lambda=\left(1^{m_{1}}, 2^{m_{2}}, \ldots,(n+1)^{m_{n+1}}\right)$ be an arbitrary partition consisting of parts at most equal to $n+1$. We have

$$
\begin{aligned}
P_{\lambda}(q, t)=\sum_{\theta \in \mathrm{M}^{(n+1)}} \prod_{k=1}^{n} C_{\theta_{1, k+1}, \ldots, \theta_{k, k+1}}^{(t, q)}\left(\left\{u_{i}=q^{k-i} t^{\sum_{j=i}^{k} m_{j}+\sum_{j=k+2}^{n+1}\left(\theta_{i, j}-\theta_{k+1, j}\right)} ; 1 \leq i \leq k\right\}\right) \\
\times \prod_{k=1}^{n+1} e_{\sum_{j=k}^{n+1} m_{j}+\sum_{j=k+1}^{n+1} \theta_{k j}-\sum_{j=1}^{k-1} \theta_{j k}} .
\end{aligned}
$$


It is clear that the analytic developments given above are fully explicit. It is worth considering these results in some particular cases [12, p. 324], namely $q=t$ (Schur functions), $q=1$ (elementary symmetric functions), $t=1$ (monomial symmetric functions), $q=0$ (Hall-Littlewood symmetric functions), and $q=t^{\alpha}, t \rightarrow 1$ (Jack symmetric functions). This is done in detail in [1], together with the case of hook partitions $\left(r, 1^{s}\right)$, already worked out by Kerov [4].

\section{Proof}

We shall need the two following elementary lemmas. Let $a, u=\left(u_{1}, \ldots, u_{n+1}\right)$ and $v=\left(v_{1}, \ldots, v_{n}\right)$ be $2 n+2$ indeterminates. We write $v_{n+1}=0$. Define

$$
H(u ; v)=\frac{1}{\Delta(v)} \operatorname{det}_{1 \leq i, j \leq n}\left[v_{i}^{n-j}\left(1+a t^{j} \prod_{k=1}^{n+1} \frac{u_{k}-v_{i}}{t u_{k}-v_{i}}\right)\right] .
$$

Lemma 1. (i) We have

$$
H(u, v)=\frac{1}{\Delta(u)} \operatorname{det}_{1 \leq i, j \leq n+1}\left[u_{i}^{j}\left(1+a t^{j+1} \prod_{k=1}^{n} \frac{u_{i}-v_{k}}{t u_{i}-v_{k}}\right)\right] .
$$

(ii) $H(u, v)$ satisfies the functional equation

$$
\begin{aligned}
\sum_{i=1}^{n+1} \prod_{\substack{k=1 \\
k \neq i}}^{n+1} \frac{v_{i} / t-v_{k}}{v_{i}-v_{k}} \prod_{k=1}^{n+1} \frac{1-v_{i} / u_{k}}{1-v_{i} / t u_{k}} H\left(u ; v_{1}, \ldots, v_{i} / q, \ldots, v_{n}\right)= \\
\qquad \sum_{i=1}^{n+1} \prod_{\substack{k=1 \\
k \neq i}}^{n+1} \frac{u_{k} / t-u_{i}}{u_{k}-u_{i}} \prod_{k=1}^{n+1} \frac{1-v_{k} / u_{i}}{1-v_{k} / t u_{i}} H\left(u_{1}, \ldots, q u_{i}, \ldots, u_{n+1} ; v\right) .
\end{aligned}
$$

(iii) $H(u, v)$ satisfies the functional equation

$$
\begin{aligned}
\sum_{i=1}^{n+1} \prod_{\substack{k=1 \\
k \neq i}}^{n+1} \frac{t v_{i}-v_{k}}{v_{i}-v_{k}} \prod_{k=1}^{n+1} \frac{1-q v_{i} / t u_{k}}{1-q v_{i} / u_{k}} H\left(u ; v_{1}, \ldots, q v_{i}, \ldots, v_{n}\right)= \\
\qquad \sum_{i=1}^{n+1} \prod_{\substack{k=1 \\
k \neq i}}^{n+1} \frac{t u_{k}-u_{i}}{u_{k}-u_{i}} \prod_{k=1}^{n+1} \frac{1-q v_{k} / t u_{i}}{1-q v_{k} / u_{i}} H\left(u_{1}, \ldots, u_{i} / q, \ldots, u_{n+1} ; v\right) .
\end{aligned}
$$

Proof. Consider the indeterminates $X=\left(x_{1}, \ldots, x_{n+1}\right)$ and $Y=\left(y_{1}, \ldots, y_{n+1}\right)$, with $x_{i}=v_{i}$ and $y_{i}=1 / u_{i}$. Writing $\Pi$ for $\Pi(X, Y ; 1 / q, 1 / t)$ we have obviously

$$
\begin{gathered}
\Pi^{-1} T_{1 / q, x_{i}} \Pi=\prod_{k=1}^{n+1} \frac{1-v_{i} / u_{k}}{1-v_{i} / t u_{k}} \quad, \quad \Pi^{-1} T_{1 / q, y_{i}} \Pi=\prod_{k=1}^{n+1} \frac{1-v_{k} / u_{i}}{1-v_{k} / t u_{i}} \\
H(u ; v)=\Pi^{-1} D(a ; 1 / q, 1 / t)_{(X)} \Pi
\end{gathered}
$$


Taking into account (1), we deduce (i) immediately. We have also

$$
\begin{aligned}
& \Pi^{-1} T_{1 / q, x_{i}} D(a ; 1 / q, 1 / t)_{(X)} \Pi=\prod_{k=1}^{n+1} \frac{1-v_{i} / u_{k}}{1-v_{i} / t u_{k}} H\left(u ; v_{1}, \ldots, v_{i} / q, \ldots, v_{n}\right), \\
& \Pi^{-1} T_{1 / q, y_{i}} D(a ; 1 / q, 1 / t)_{(Y)} \Pi=\prod_{k=1}^{n+1} \frac{1-v_{k} / u_{i}}{1-v_{k} / t u_{i}} H\left(u_{1}, \ldots, q u_{i}, \ldots, u_{n+1} ; v\right),
\end{aligned}
$$

and (ii) follows from (2). Finally

$$
\Pi^{-1} T_{q, x_{i}} \Pi=\prod_{k=1}^{n+1} \frac{1-q v_{i} / t u_{k}}{1-q v_{i} / u_{k}} \quad, \quad \Pi^{-1} T_{q, y_{i}} \Pi=\prod_{k=1}^{n+1} \frac{1-q v_{k} / t u_{i}}{1-q v_{k} / u_{i}},
$$

This implies

$$
\begin{aligned}
& \Pi^{-1} T_{q, x_{i}} D(a ; 1 / q, 1 / t)_{(X)} \Pi=\prod_{k=1}^{n+1} \frac{1-q v_{i} / t u_{k}}{1-q v_{i} / u_{k}} H\left(u ; v_{1}, \ldots, q v_{i}, \ldots, v_{n}\right), \\
& \Pi^{-1} T_{q, y_{i}} D(a ; 1 / q, 1 / t)_{(Y)} \Pi=\prod_{k=1}^{n+1} \frac{1-q v_{k} / t u_{i}}{1-q v_{k} / u_{i}} H\left(u_{1}, \ldots, u_{i} / q, \ldots, u_{n+1} ; v\right),
\end{aligned}
$$

and (iii) follows from (3).

Being given a partition $\lambda=\left(\lambda_{1}, \ldots, \lambda_{n}\right)$ and $1 \leq k \leq n$, we denote $\lambda_{(k)}=\left(\lambda_{1}, \ldots, \lambda_{k}-\right.$ $\left.1, \ldots, \lambda_{n}\right)$.

Lemma 2. Let $X=\left(x_{1}, \ldots, x_{N}\right)$ and $z$ be $N+1$ indeterminates and $\lambda=\left(\lambda_{1}, \ldots, \lambda_{n}\right)$ an arbitrary partition with length $n \leq N$. The coefficient of $z$ in $Q_{\lambda}(X, z ; q, t)$ is given by

$$
\frac{1-t}{1-q} \sum_{k=1}^{n} c_{k}(\lambda) Q_{\lambda_{(k)}}(X ; q, t)
$$

with $c_{k}(\lambda)=0$ if $\lambda_{(k)}$ is not a partition, and otherwise

$$
c_{k}(\lambda)=\prod_{i=1}^{k-1} \frac{1-q^{\lambda_{i}-\lambda_{k}+1} t^{k-i-1}}{1-q^{\lambda_{i}-\lambda_{k}+1} t^{k-i}} \frac{1-q^{\lambda_{i}-\lambda_{k}} t^{k-i+1}}{1-q^{\lambda_{i}-\lambda_{k}} t^{k-i}} .
$$

Proof. We have

$$
Q_{\lambda}(X, z)=\sum_{\mu} Q_{\lambda / \mu}(X) Q_{\mu}(z)
$$

where $Q_{\lambda / \mu}$ denotes the skew Macdonald function [12, p. 345]. But $Q_{\mu}(z)$ is non zero only if $\mu$ is a row partition $(k)$, in which case $Q_{(k)}(z)=g_{k}(z)=a_{k} z^{k}$ for some constant $a_{k}$. 
In particular $a_{1}=(1-t) /(1-q)$. Thus the coefficient of $z$ in $Q_{\lambda}(X, z)$ is $(1-t) /(1-$ q) $Q_{\lambda /(1)}(X)$. Now using Macdonald's notations, the skew function $Q_{\lambda /(1)}$ is defined by

$$
Q_{\lambda /(1)}=\sum_{\nu} \psi_{\lambda / \nu}^{\prime} Q_{\nu}
$$

with $\psi_{\lambda / \nu}^{\prime}$ given by

$$
e_{1} P_{\nu}=\sum_{\lambda} \psi_{\lambda / \nu}^{\prime} P_{\lambda}
$$

It is the simplest case of the Pieri formula for Macdonald polynomials. The quantities $\psi_{\lambda / \nu}^{\prime}$ have been computed in [12], p. 336. The sum above is restricted to partitions $\nu=\lambda_{(k)}=\left(\lambda_{1}, \ldots, \lambda_{k}-1, \ldots, \lambda_{n}\right)$ for $1 \leq k \leq n$, and we have $\psi_{\lambda / \lambda_{(k)}}^{\prime}=c_{k}(\lambda)$.

Proof of Theorem 1. We start from the Pieri formula proved in [12, p. 340, (6.24), (ii)]. Being given a partition $\mu=\left(\mu_{1}, \ldots, \mu_{n}\right)$ with length $n$, this Pieri formula reads

$$
Q_{(r)} Q_{\mu}=\sum_{\kappa \supset \mu} \psi_{\kappa / \mu} Q_{\kappa}
$$

where the skew diagram $\kappa-\mu$ is a horizontal $r$ - strip, i.e. has at most one square in each column. Since $\kappa-\mu$ is a horizontal strip, the length of $\kappa$ is at most equal to $n+1$, and we can write

$$
Q_{(r)} Q_{\left(\mu_{1}, \ldots, \mu_{n}\right)}=\sum_{\theta \in \mathrm{N}^{n}} \psi_{\theta_{1}, \ldots, \theta_{n}}(\mu) Q_{\left(\mu_{1}+\theta_{1}, \ldots, \mu_{n}+\theta_{n}, r-|\theta|\right)} .
$$

Now let $\lambda=\left(\lambda_{1}, \ldots, \lambda_{n+1}\right)$ be a partition with length $n+1$. Expanding $Q_{\left(\lambda_{n+1}\right)} Q_{\left(\lambda_{1}, \ldots, \lambda_{n}\right)}$ and using induction on $\lambda_{n+1}$, the Pieri formula can be inverted as

$$
Q_{\left(\lambda_{1}, \ldots, \lambda_{n+1}\right)}=\sum_{\theta \in \mathbb{N}^{n}} F_{\theta_{1}, \ldots, \theta_{n}}\left(\lambda_{1}, \ldots, \lambda_{n+1}\right) Q_{\left(\lambda_{n+1}-|\theta|\right)} Q_{\left(\lambda_{1}+\theta_{1}, \ldots, \lambda_{n}+\theta_{n}\right)},
$$

with $F_{\theta}\left(\lambda_{1}, \ldots, \lambda_{n+1}\right)$ to be determined.

We can write this relation at $(X, z)$, apply Lemma 2 , and identify the coefficient of $z$ on each side, up to the factor $(1-t) /(1-q)$. The coefficient on the left-hand side is

$$
\begin{array}{r}
\sum_{k=1}^{n+1} c_{k}(\lambda) Q_{\lambda_{(k)}}=\sum_{\theta \in \mathbb{N}^{n}}\left(\sum_{k=1}^{n} c_{k}(\lambda) F_{\theta_{1}, \ldots, \theta_{n}}\left(\lambda_{(k)}\right) Q_{\left(\lambda_{n+1}-|\theta|\right)} Q_{\left(\lambda_{1}+\theta_{1}, \ldots, \lambda_{k}+\theta_{k}-1, \ldots, \lambda_{n}+\theta_{n}\right)}\right. \\
\left.+c_{n+1}(\lambda) F_{\theta_{1}, \ldots, \theta_{n}}\left(\lambda_{(n+1)}\right) Q_{\left(\lambda_{n+1}-|\theta|-1\right)} Q_{\left(\lambda_{1}+\theta_{1}, \ldots, \lambda_{n}+\theta_{n}\right)}\right) .
\end{array}
$$

The coefficient on the right-hand side is

$$
\begin{aligned}
\sum_{\theta \in \mathrm{N}^{n}} F_{\theta_{1}, \ldots, \theta_{n}}(\lambda) & \left(Q_{\left(\lambda_{n+1}-|\theta|-1\right)} Q_{\left(\lambda_{1}+\theta_{1}, \ldots, \lambda_{n}+\theta_{n}\right)}\right. \\
& \left.+\sum_{k=1}^{n} c_{k}\left(\left(\lambda_{1}+\theta_{1}, \ldots, \lambda_{n}+\theta_{n}\right)\right) Q_{\left(\lambda_{n+1}-|\theta|\right)} Q_{\left(\lambda_{1}+\theta_{1}, \ldots, \lambda_{k}+\theta_{k}-1, \ldots, \lambda_{n}+\theta_{n}\right)}\right) .
\end{aligned}
$$


Now the products $Q_{(r)} Q_{\mu}$ form a basis of Sym. Identifying coefficients of $Q_{\left(\lambda_{n+1}-|\theta|-1\right)}$ $Q_{\left(\lambda_{1}+\theta_{1}, \ldots, \lambda_{n}+\theta_{n}\right)}$ on both sides, we obtain

$$
\begin{aligned}
& \sum_{k=1}^{n} c_{k}(\lambda) F_{\theta_{1}, \ldots, \theta_{k}+1, \ldots, \theta_{n}}\left(\lambda_{(k)}\right)+c_{n+1}(\lambda) F_{\theta_{1}, \ldots, \theta_{n}}\left(\lambda_{(n+1)}\right)= \\
& F_{\theta_{1}, \ldots, \theta_{n}}(\lambda)+\sum_{k=1}^{n} c_{k}\left(\left(\lambda_{1}+\theta_{1}, \ldots, \lambda_{k}+\theta_{k}+1, \ldots, \lambda_{n}+\theta_{n}\right)\right) F_{\theta_{1}, \ldots, \theta_{k}+1, \ldots, \theta_{n}}(\lambda) .
\end{aligned}
$$

Here we should keep in mind that this relation keeps true when some $\theta_{i}$ are negative, in which case the corresponding $F_{\theta}(\lambda)$ is of course zero.

An essential fact is that this equation can be used to define $F_{\theta_{1}, \ldots, \theta_{n}}(\lambda)$ by induction. If $\theta$ has some vanishing components, say $\theta_{i}=0$ for $i \in I$, we have at once

$$
c_{k}\left(\left(\lambda_{1}+\theta_{1}, \ldots, \lambda_{n}+\theta_{n}\right)\right) F_{\theta}(\lambda)=c_{k}(\lambda) F_{\theta}\left(\lambda_{(k)}\right),
$$

for any $k \notin I$. An induction on $|\lambda|$ is then sufficient to define $F_{\theta}(\lambda)$. If $\theta$ has no vanishing component, three induction steps are needed, first on $|\lambda|$, then on $|\theta|$, and finally on $\theta_{n}$. Indeed for $|\lambda|=p,|\theta|=q, \theta_{n}=r$, equation (4) defines $c_{k}\left(\left(\lambda_{1}+\theta_{1}, \ldots, \lambda_{n}+\theta_{n}\right)\right) F_{\theta}(\lambda)$ as a sum of contributions $F_{\kappa}(\mu)$, with either $|\mu|=|\lambda|-1$, either $|\kappa|=q-1$ or $\kappa_{n}=p-1$.

Moreover since $c_{k}(\lambda)$ depends only on the quantities $q^{\lambda_{i}-\lambda_{j}}$, and not on $q^{\lambda_{n+1}}$, the induction (4) implies that $F_{\theta_{1}, \ldots, \theta_{n}}(\lambda)$ depends only on the quantities $q^{\lambda_{i}-\lambda_{n+1}}, 1 \leq i \leq n$. So we may write

$$
F_{\theta_{1}, \ldots, \theta_{n}}\left(\lambda_{1}, \ldots, \lambda_{n+1}\right)=F_{\theta_{1}, \ldots, \theta_{n}}\left(u_{1}, \ldots, u_{n}\right),
$$

with $u_{i}=q^{\lambda_{i}-\lambda_{n+1}} t^{n-i}, 1 \leq i \leq n$. Setting $v_{i}=q^{\theta_{i}} u_{i}$, the recurrence relation (4) may be written

$$
\begin{gathered}
\sum_{k=1}^{n} \prod_{i=1}^{k-1} \frac{1-q u_{i} / t u_{k}}{1-q u_{i} / u_{k}} \frac{1-t u_{i} / u_{k}}{1-u_{i} / u_{k}} F_{\theta_{1}, \ldots, \theta_{k}+1, \ldots, \theta_{n}}\left(u_{1}, \ldots, u_{k} / q, \ldots, u_{n}\right) \\
+\prod_{i=1}^{n} \frac{1-q u_{i}}{1-q t u_{i}} \frac{1-t^{2} u_{i}}{1-t u_{i}} F_{\theta_{1}, \ldots, \theta_{n}}(q u)= \\
F_{\theta_{1}, \ldots, \theta_{n}}(u)+\sum_{k=1}^{n} \prod_{i=1}^{k-1} \frac{1-v_{i} / t v_{k}}{1-v_{i} / v_{k}} \frac{1-t v_{i} / q v_{k}}{1-v_{i} / q v_{k}} F_{\theta_{1}, \ldots, \theta_{k}+1, \ldots, \theta_{n}}(u) .
\end{gathered}
$$

By the inductive hypothesis, the property being obvious for $|\theta|=0$, it remains only to show that $c_{\theta_{1}, \ldots, \theta_{n}}^{(q, t)}(u ; v)$ satisfies this equation. Using

$$
\begin{aligned}
& c_{\theta_{1}, \ldots, \theta_{n}}^{(q, t)}(u ; v)= \\
& \left.\quad \prod_{k=1}^{n} t^{\theta_{k}} \frac{(q / t ; q)_{\theta_{k}}}{(q ; q)_{\theta_{k}}} \frac{\left(q u_{k} ; q\right)_{\theta_{k}}}{\left(q t u_{k} ; q\right)_{\theta_{k}}} \prod_{1 \leq i<j \leq n} \frac{\left(q u_{i} / t u_{j} ; q\right)_{\theta_{i}}}{\left(q u_{i} / u_{j} ; q\right)_{\theta_{i}}} \frac{\left(t u_{i} / v_{j} ; q\right)_{\theta_{i}}}{\left(u_{i} / v_{j} ; q\right)_{\theta_{i}}} H(u, 1 / t ; v)\right|_{a=-1},
\end{aligned}
$$


after an easy computation, it is sufficient to prove

$$
\begin{aligned}
\sum_{k=1}^{n} \frac{1-u_{k}}{1-t u_{k}} \prod_{\substack{i=1 \\
i \neq k}}^{n} \frac{u_{k} / t-u_{i}}{u_{k}-u_{i}} \prod_{i=1}^{n} \frac{t u_{k}-q v_{i}}{u_{k}-q v_{i}} H\left(u_{1}, \ldots, u_{k} / q, \ldots, u_{n}, 1 / t ; v\right) \\
+\prod_{i=1}^{n} \frac{1-t^{2} u_{i}}{1-t u_{i}} \frac{1-q v_{i}}{1-q t v_{i}} H(q u, 1 / t ; q v)=H(u, 1 / t ; v)+ \\
\quad \sum_{k=1}^{n} \frac{1-q v_{k}}{1-q t v_{k}} \prod_{\substack{i=1 \\
i \neq k}}^{n} \frac{v_{k}-v_{i} / t}{v_{k}-v_{i}} \prod_{i=1}^{n} \frac{t u_{i}-q v_{k}}{u_{i}-q v_{k}} H\left(u, 1 / t ; v_{1}, \ldots, q v_{k}, \ldots, v_{n}\right) .
\end{aligned}
$$

Since $H\left(u, u_{n+1} / q ; v\right)=H\left(q u, u_{n+1} ; q v\right)$ we conclude by applying Lemma 1 (iii), with $u_{n+1}=1 / t$ and $v_{n+1}=0$.

Remark : Starting from Lemma 1 (ii), the same argument applied in reverse order shows that $F_{\theta_{1}, \ldots, \theta_{n}}$ also satisfies the following recurrence relation

$$
\begin{aligned}
& \sum_{k=1}^{n} \prod_{i=k+1}^{n} \frac{1-v_{k} / t v_{i}}{1-v_{k} / v_{i}} \frac{1-t v_{k} / q v_{i}}{1-v_{k} / q v_{i}} F_{\theta_{1}, \ldots, \theta_{k}-1, \ldots, \theta_{n}}(u)+F_{\theta_{1}, \ldots, \theta_{n}}(u)=F_{\theta_{1}, \ldots, \theta_{n}}(u / q)+ \\
& \sum_{k=1}^{n} \frac{1-q u_{k}}{1-q t u_{k}} \frac{1-t^{2} u_{k}}{1-t u_{k}} \prod_{i=k+1}^{n} \frac{1-q u_{k} / t u_{i}}{1-q u_{k} / u_{i}} \frac{1-t u_{k} / u_{i}}{1-u_{k} / u_{i}} F_{\theta_{1}, \ldots, \theta_{k}-1, \ldots, \theta_{n}}\left(u_{1}, \ldots, q u_{k}, \ldots, u_{n}\right) .
\end{aligned}
$$

However this second relation seems much more difficult to obtain than (5).

\section{References}

[1] L.-K. Hua, Harmonic analysis of functions of several complex variables in the classical domains, American Mathematical Society, Providence, 1963.

[2] A. T. James, Zonal polynomials of the real positive definite symmetric matrices, Ann. Math., 74 (1961), 456-469.

[3] N. H. Jing, T. Józefiak, A formula for two-row Macdonald functions, Duke Math. J., 67 (1992), 377-385.

[4] S. Kerov, Generalized Hall-Littlewood symmetric functions and orthogonal polynomials, Adv. Sov. Math., Amer. Math. Soc., Providence, R.I., 9 (1992), 67-94.

[5] C. Krattenthaler, Operator methods and Lagrange inversion, a unified approach to Lagrange formulas, Trans. Amer. Math. Soc., 305 (1988), 431-465.

[6] C. Krattenthaler, A new matrix inverse, Proc. Amer. Math. Soc., 124 (1996), 47-59. 
[7] L. Lapointe, A. Lascoux, J. Morse, Determinantal expressions for Macdonald polynomials, Int. Math. Res. Not., 18 (1998), 957-978.

[8] M. Lassalle, Explicitation des polynômes de Jack et de Macdonald en longueur trois, C. R. Acad. Sci. Paris Sér. I Math., 333 (2001), 505-508.

[9] M. Lassalle, Une q - spécialisation pour les fonctions symétriques monomiales, Adv. Math., 162 (2001), 217-242.

[10] M. Lassalle and M. Schlosser, An analytic formula for Macdonald polynomials, C. R. Acad. Sci. Paris, 337 (2003), 569-574.

[11] M. Lassalle and M. Schlosser, Inversion of the Pieri formula for Macdonald polynomials, http://www.arxiv.org/abs/math.CO/0402127.

[12] I. G. Macdonald, Symmetric functions and Hall polynomials, Clarendon Press, second edition, Oxford, 1995.

[13] M. Schlosser, Multidimensional matrix inversions and $A_{r}$ and $D_{r}$ basic hypergeometric series, Ramanujan J., 1 (1997), 243-274. 